\title{
Query by Image Content using Color-Texture Features Extracted from Haar Wavelet Pyramid
}

\author{
Dr.H.B.Kekre \\ Senior Professor \\ Computer Engineering Department, \\ MPSTME, NMIMS (Deemed-to-be \\ University), Mumbai, India
}

\author{
Sudeep D. Thepade \\ Ph.D.Research Scholar \& Associate. \\ Professor \\ Computer Engineering Department, \\ MPSTME, NMIMS (Deemed-to-be \\ University), Mumbai, India
}

\author{
Akshay Maloo \\ B.Tech (CE) Student \\ Computer Engineering Department, \\ MPSTME,NMIMS (Deemed-to-be \\ University), Mumbai, India
}

\begin{abstract}
The paper presents the Wavelet Pyramid based image retrieval techniques [1] using Haar transform. Here content based image retrieval (CBIR) is done using the image feature set extracted from Haar Wavelets applied on the image at various levels of decomposition. Here the database image features are extracted by applying Haar Wavelets on gray plane (average of red, green and blue) and color planes (red, green and blue components). The techniques Gray-Haar Wavelets and Color-Haar Wavelets are tested on image database having 11 categories with total 1000 images. Total 55 queries are fired on the database. The results show that precision and recall of Haar Wavelets are better than complete Haar transform based CBIR, which proves that Haar Wavelets gives better discrimination capability in image retrieval at higher query execution speed, per higher level Haar Wavelets. Color-Haar Wavelets based CBIR have greater precision and recall than Gray-Haar Wavelets based CBIR. The Haar Wavelets level-5 outperforms other Haar Wavelets, because the higher level Haar Wavelets are giving very coarse color-texture features while the lower level are representing very fine color-texture features which are less useful to differentiate the images in image retrieval.
\end{abstract}

\section{Keywords}

Content Based Image Retrival (CBIR), Haar Wavelets, Haar wavelet Pyramid, Color-texture

\section{INTRODUCTION}

The large numbers of image collections have posed increasing technical challenges to computer systems to store/transmit and manage image data effectively [3]. The storage and transmission challenge is tackled by image compression [14]. The challenge to retrieve stored images is studied in the context of image database [13], and has been attempted by researchers worldwide from a wide range of disciplines from computer vision [15], image processing [16]-[18] to traditional database areas for over a decade [20],[22]. Researchers are discovering that the process of locating a desired image in a large and varied collection can be a source of considerable frustration [2],[19],[26]. Problems with traditional methods of image indexing [1]-[3],[20] have led to the rise of interest in techniques for retrieving images on the basis of automatically-derived features such as colour, texture and shape a technology now generally referred to as Content-Based Image Retrieval (CBIR) [23], [27]. After a decade of intensive research, CBIR technology is now beginning to move out of the laboratory and into the marketplace, in the form of commercial products like QBIC [18] and Virage [17]. However, the technology still lacks maturity, and is not yet being used on a significant scale [3]. In the absence of hard evidence on the effectiveness of CBIR in practice, opinion is still sharply divided about their usefulness in handling real-life queries in large and diverse image databases/collections [15]. A wide range of possible applications for CBIR technology has been identified [3],[13]. There are mainly two streams of research for image retrieval. The database community is focusing on image indexing [21] whereas image processing groups are concentrating on representing the image content in the form of some feature descriptors [16]. Most of the current image indexing practices mainly rely on color, texture or shape features. The performance of the image retrieval technique improves if these features are combined and considered together [3]. In case of color and texture features combination, the red, green and blue planes are considered separately and then some texture features are extracted from these color planes. The limitation of extracting color-texture features is the fine-tuning of these features [24].

A wide variety of techniques for describing image texture have been proposed. Tuceryan and Jain [28] divided texture analysis methods into four categories: statistical, model-based, geometrical and signal processing. Here we have considered only signal processing for texture extraction and representation. If texture features are too coarse or too fine, they cannot help much in image discrimination, so the size of feature vector plays important role in performance of image retrieval [25]. Also the speed of retrieval is important aspect in image retrieval. Here theWavelet Pyramid based image retrieval techniquesdiscussed in [1] are presented using Haar transform and the performances of respective methods are tested and discussed.

The section 2 discusses the Haar transform. Section 3 gives steps to generate Haar Wavelets of different levels from an image. Section 4 has the concept of Haar wavelet pyramid. Section 5 takes quick preliminary overview of CBIR. Section 6 gives the proposed image retrieval techniques. The implementation and results are discussed respectively in sections 7 and 8 . The conclusions are given in section 9.

\section{HAAR TRANSFORM}

This sequence was proposed in 1909 by AlfrédHaar [9]. Haar used these functions to give an example of a countable orthonormal system for the space of square-integrable functions on the real line. The study of Wavelets, and even the term "wavelet", did not come until much later [10]. The Haar wavelet is also the simplest possible wavelet. The technical disadvantage of the Haar wavelet is that it is not continuous, and therefore not 
differentiable. This property can, however, be an advantage for the analysis of signals with sudden transitions, such as monitoring of tool failure in machines.

The Haar wavelet's mother wavelet function $\psi(\mathrm{t})$ can be described as:

$$
\psi(t)=\left\{\begin{aligned}
1, & 0 \leq t<\frac{1}{2} \\
-1 & , \frac{1}{2} \leq t<1 \\
0, & \text { otherwise }
\end{aligned}\right.
$$

and its scaling function $\varphi(t)$ can be described as:

$$
\varphi(t)=\left\{\begin{array}{l}
1,0 \leq t<1 \\
0, \text { otherwise }
\end{array}\right.
$$

\section{HAAR WAVELETS}

The procedure of generating Haar Wavelets $[1,6,7]$ is shown in flowchart given in figure 1 and can be explained as given in following steps. Let $\mathrm{I}_{\mathrm{NXN}}$ be the image with size $\mathrm{NxN}$ of which Haar Wavelets are to be obtained and $\mathrm{H}_{\mathrm{NxN}}$ be the Haar transform matrix of size $\mathrm{NxN}$.

a. Apply Haar transform of size $\mathrm{NxN}$ on the image of size $\mathrm{NxN}$ to get Haar transformed image with approximation (hIA), horizontal (hIH), vertical (hIV) and diagonal (hID) components.

$$
\begin{aligned}
\mathrm{hI}_{\mathrm{NxN}} & =[\mathrm{hIA}, \mathrm{hIH}, \mathrm{hIV}, \mathrm{hID}] \\
& =\left[\mathrm{H}_{\mathrm{NxN}}\right]\left[\mathrm{I}_{\mathrm{NxN}}\right]\left[\mathrm{H}^{\prime}{ }_{\mathrm{NxN}}\right]
\end{aligned}
$$

b. Replace horizontal (hIH), vertical (hIV) and diagonal (hID) components with zero to get modified Haar transformed image 'hwI'.

$$
\text { hwI } \mathrm{I}_{\mathrm{NxN}}=[\mathrm{hIA}, \text { Zero, Zero, Zero }]
$$

c. Apply inverse Haar transform on the modified Haar transformed image to get h'wI.

$$
h^{\prime} \mathrm{wI}_{\mathrm{NxN}}=\left[\mathrm{H}^{\prime}{ }_{\mathrm{NxN}}\right]\left[\mathrm{hwI} \mathrm{I}_{\mathrm{NxN}}\right]\left[\mathrm{H}_{\mathrm{NxN}}\right]
$$

d. Down-sample the result of step'c' (h'wI) by taking alternate rows and columns to get imagewith size $\mathrm{N} / 2 \times \mathrm{N} / 2$.

$$
\left.\mathrm{dhI}_{\mathrm{N} / 2 \times \mathrm{N} / 2}=\text { downsample( h' } \mathrm{wI}_{\mathrm{NxN}}\right)
$$

e. Apply Haar transform of size $\mathrm{N} / 2 \mathrm{xN} / 2$ on downsampled image $\left(\mathrm{dwI}_{\mathrm{N} / 2 \times \mathrm{N} / 2}\right)$ to get theHaar Wavelet of level-1.

Haar Wavelet Level I =

$$
\left[\mathrm{W}_{\mathrm{N} / 2 \times \mathrm{N} / 2}\right]\left[\mathrm{dwI} \mathrm{I}_{\mathrm{N} / 2 \times \mathrm{N} / 2}\right]\left[\mathrm{W}^{\prime}{ }_{\mathrm{N} / 2 \times \mathrm{N} / 2}\right]
$$

f. Repeat steps b to e 'P-1' times on the level 1Haar Wavelet to get Haar Wavelet of level 'P'.

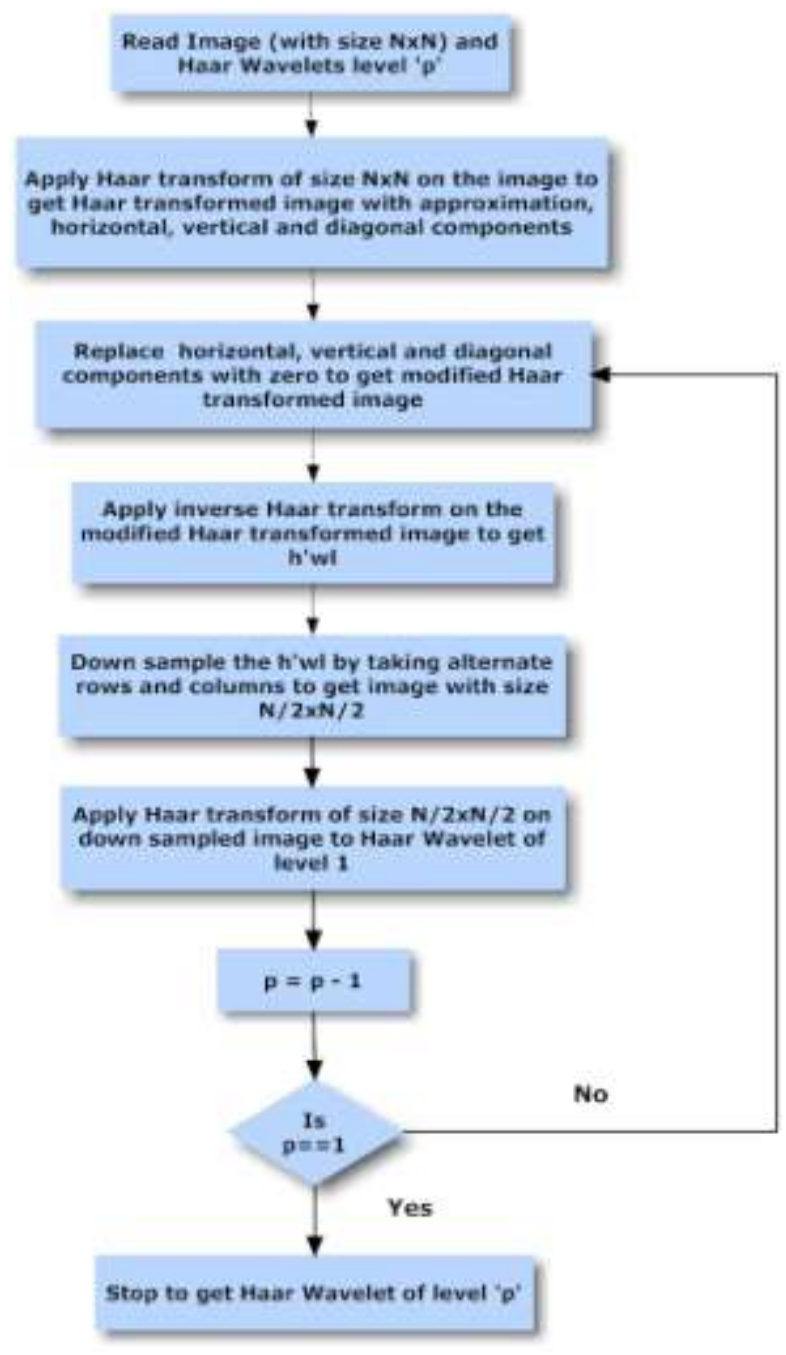

Figure1. Flowchart for generating Haar Wavelets of level 'p'

\section{HAAR WAVELET PYRAMID}

The Haar Wavelets of particular image at different levels, when considered together gives Haar Wavelet Pyramid [1]. Here for the first level of Haar Wavelet pyramid, Haar transform is applied on the image to get approximation, horizontal, vertical and diagonal components. The approximation components of first level Haar Wavelet is considered to be transformed with Haar T. to get second level Haar Wavelet. The Haar Wavelet pyramid of a sample images are shown in figure 2 given below. Where the baby image is decomposed into three levels of Haar Wavelet pyramid as Haar Wavelet level-1, Haar Wavelet level-2 and Haar Wavelets level-3. 


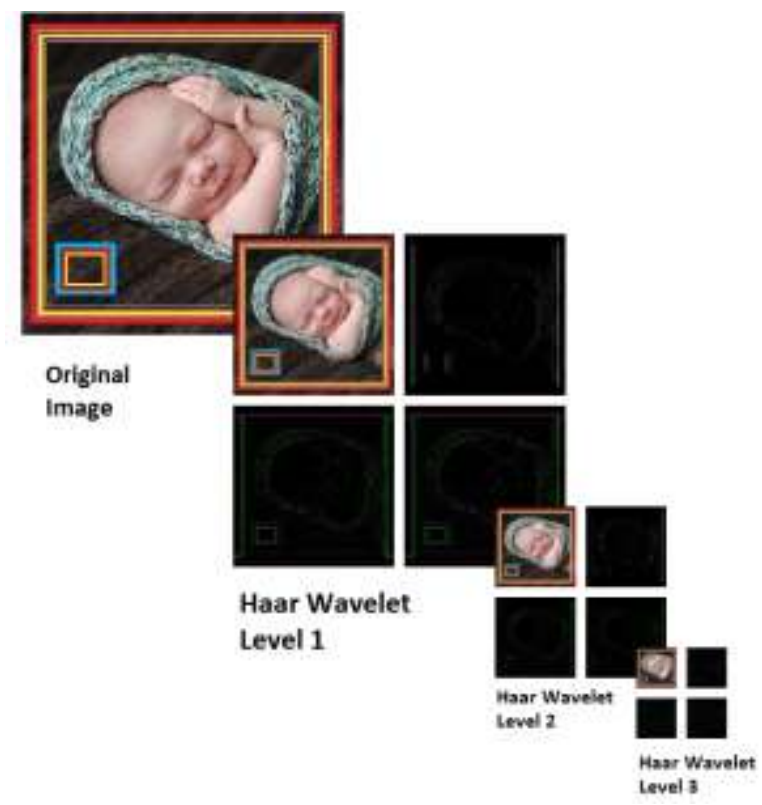

Figure2.Different Levels of Haar Wavelet Pyramid

\section{CONTENT BASED IMAGE RETRIEVAL}

The earliest use of the term content-based image retrieval in the literature seems to have been by Kato et.al.[8], to describe his experiments into automatic retrieval of images from a database by color and shape feature. The term has since been widely used to describe the process of retrieving images from a large collection on the basis of features (such as color, shape andtexture) that can be automatically extracted from images themselves.

The typical CBIR system performs two major tasks [1],[5]. The first one is feature extraction (FE), where a set of features, called image signature or feature vector, is generated to represent the content of each image in the database. A feature vector is much smaller in size than the original image, typically of the order of hundreds of elements (rather than millions). The second task of CBIR system is similarity measurement (SM) Here a distance between the query image and each image in the database using their signatures is computed so that the top "closest" images can be retrieved.

Most commonly Euclidian distance and correlation coefficient [9],[10] are used as similarity measure in CBIR. Correlation coefficient measures the cosine of the angle between two vectors and varies from 0 to 1 . When it is 1 both the vectors are aligned but their magnitude may not be same. In contrast to this Euclidian measure gives the distance between the vectors, when it is 0 not only the vectors are aligned but their magnitude is also same. For this reason we have preferred Euclidian distance as a similarity measure.

The direct Euclidian distance between an image $\mathrm{P}$ and query image Q can be given as below.

$$
E D=\sqrt{\sum_{i=1}^{n}(V p i-V q i)^{2}}
$$

where, Vpi and Vqi be the feature vectors of image P and Query image $Q$ respectively with size ' $n$ '.

\section{IMAGE RETRIEVAL USING HAAR WAVELETS PYRAMID}

\subsection{Feature Extraction}

Here the approximate components of Haar Wavelet level-1, Haar Wavelet level-2,...,Haar Wavelet level-7 are obtained for every image in the database and haar transforms of respective sizes are applied on them, the results are stored as feature vectors for respective image. At level-1 Haar Wavelet the feature vector size is $\mathrm{N} / 2 \mathrm{xN} / 2$. At level-2 Haar Wavelet the feature vector size is $\mathrm{N} / 4 \mathrm{xN} / 4$ and so on. Thus the feature vectors for upto level-7 Haar Wavelets are extracted and the feature vector database is generated.

The Gray-Haar Wavelets are extracted from gray images (average of red, green and blue components is taken as gray). Then the Haar Wavelets of Red, Green and Blue planes of images are extracted and considered as Color-Haar Wavelets of respective images for various levels.

\subsection{Query Execution}

Here the feature set of Haar Wavelet level-p is extracted as a feature set for query image using proposed technique of Haar Wavelet generation. Then these are matched with Haar Wavelet level-p feature vector database using Euclidian distance as similarity measure. As compared to applying complete Haar transform on the image, this proposed method takes fewer computations to extract the feature set and gives better precision and recall values.

For image of size $\mathrm{NxN}$ complete Haar needs $2 \mathrm{~N}^{2} \log _{2}(\mathrm{~N})$ additions and for Haar Wavelet of level-p the number of additions needed are $2\left(\mathrm{~N} / 2^{\mathrm{p}}\right)^{2} \log \left(\mathrm{N} / 2^{\mathrm{p}}\right)$ as the size of feature vector would be $\left(\mathrm{N} / 2^{\mathrm{p}}\right) \mathrm{x}\left(\mathrm{N} / 2^{\mathrm{p}}\right)$. This gives tremendous reduction in query execution time using higher Haar Wavelet level.

\section{IMPLEMENTATION}

The method is implemented in Matlab 7.0 on Intel Core 2 Duo Processor T8100, $2.1 \mathrm{GHz}, 2$ GB RAM machine to obtain results. To check the performance of proposed technique we have used precision and recall. To test the proposed CBIR techniques using Haar Wavelets, we have used databases database [11] of 1000 variable size images spread across 11 categories of human being, animals, natural scenery and manmade things. The sample images from the database are shown in figure 3 . Here all images were resized to $256 \times 256 \times 3$ before using Haar Wavelets for feature extraction. Five queries were selected from each category of images, so in all 55 queries for every Haar Wavelet level and complete Haar $\mathrm{T}$. are fired on the database to test the proposedCBIR techniques.

In [12] Jain et al. address some of the features of an efficient CBIR system such as accuracy, stability and speed. To measure the retrieval effectiveness, we have used the precision and recall as statistical comparison parameters for our proposed technique of CBIR. The standard definitions of these two measures are given by following equations. 


$$
\begin{gathered}
\operatorname{Pr} \text { ecision }=\frac{\text { Number_of_relevant_images_retrieved }}{\text { Total_number_of_images_retrieved }} \\
\operatorname{Re} \text { call }=\frac{\text { Number_of_relevant_images_retrieved }}{\text { Total_number_of_relevent_images_in_database }}
\end{gathered}
$$

\section{RESULTS AND DISCUSSIONS}

Figure 4 gives the results of first 10 retrieved images for a sample query image for the proposed image retrieval techniques. Figure 4.a is the queryimage, figures4.b, 4.c, 4.d and 4.e respectively shows result images for RGB-Haar Wavelets-Level-1, RGB-Haar Wavelets-Level-3, RGB-Haar Wavelets-Level-5 and RGB-Haar Wavelets-Level-7. The relevant retrieved images in figure 4 are highlighted using pink boundary. RGB-Haar Wavelets-Level-5 gives highest number of relevant images in first ten retrieved images set. The precision and recall of all queries are obtained for the feature sets of different levels Haar Wavelets. Net average precision and recall are plotted against number of retrieved images for different levels of Haar Wavelets along with complete Haar transform based CBIR as shown in figure 5 and figure 6 respectively. In figure 5 , the net average precision is plotted for proposed CBIR methods. Here Haar Wavelets are outperforming complete Haar in case of precision. The Precision/Recall values of Color-Haar Wavelets(RGB) are higher than those of Gray-Haar Wavelets. The precision values of Haar Waveletslevel-5 are higher than all other Haar Wavelets and even than the complete Haar based CBIR.

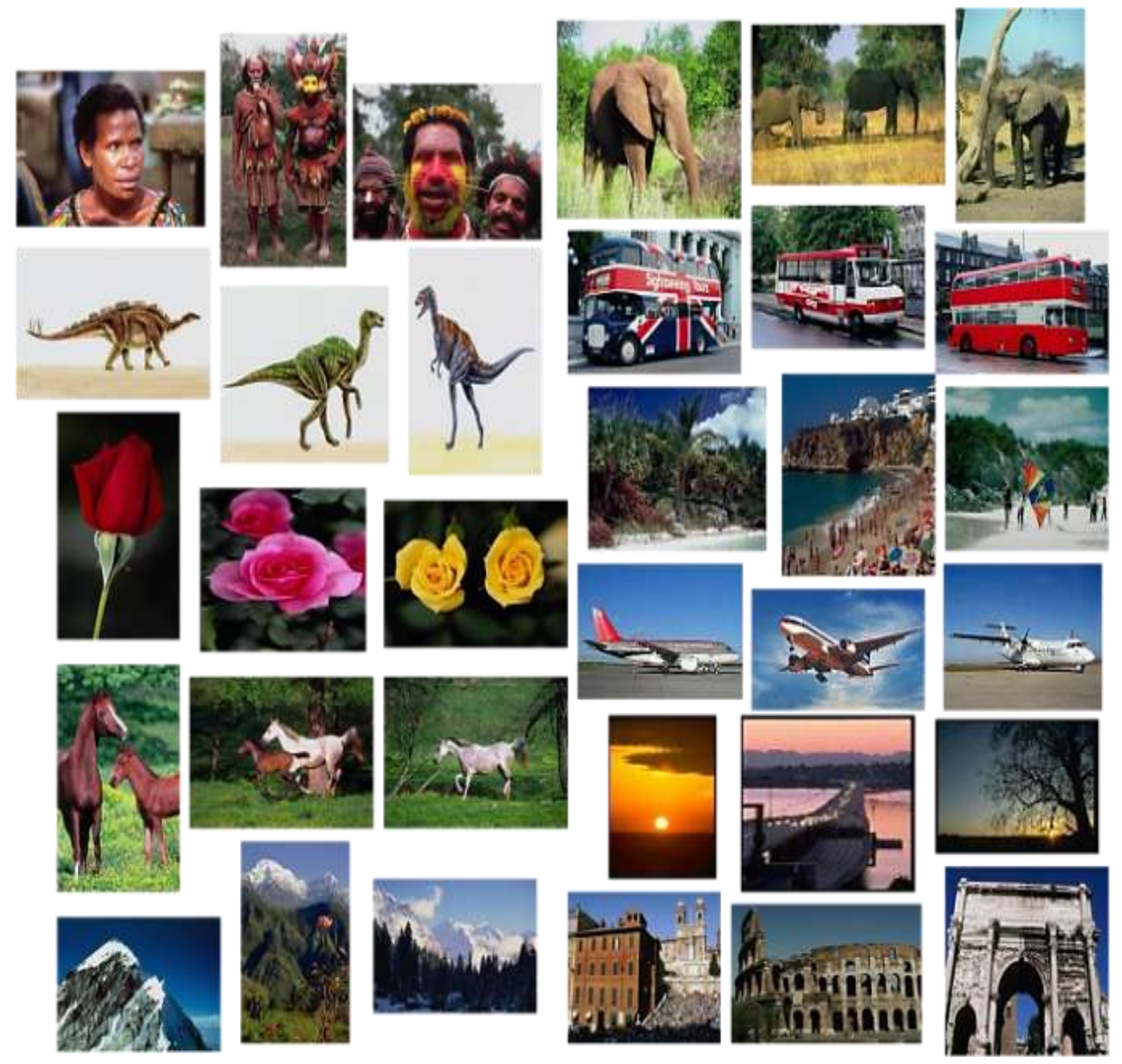

Figure. 3. Sample images from first database, the database has 11 categories, for a total of 1,000 images. 


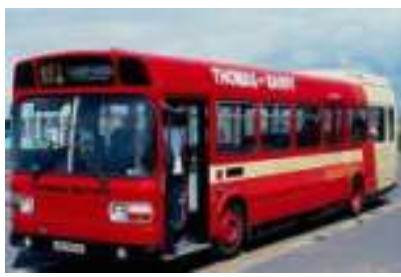

4.a. Sample Query Image from ‘Monuments' Category

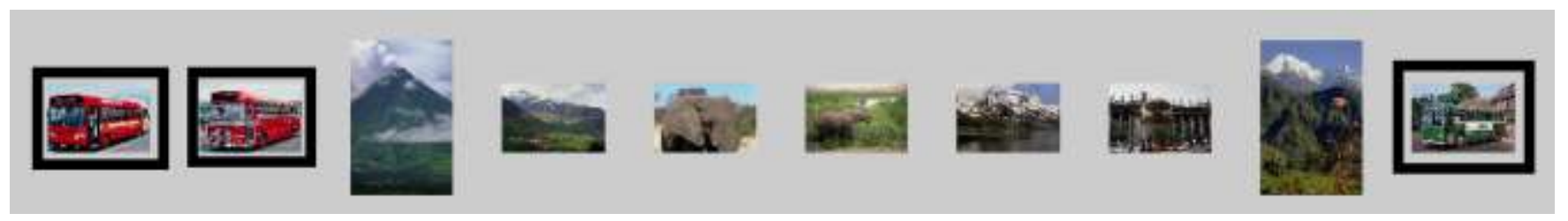

4.b.First Ten Retrieved images using RGB-Haar Wavelets-Level-1 image retrieval technique (Total Relevant retrieved images=03)

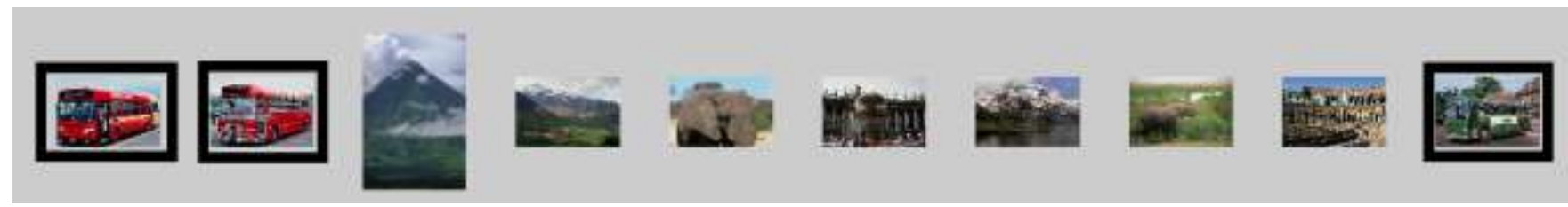

4.c.First Ten Retrieved images using RGB-Haar Wavelets-Level-3 image retrieval technique (Total Relevant retrieved images=03)

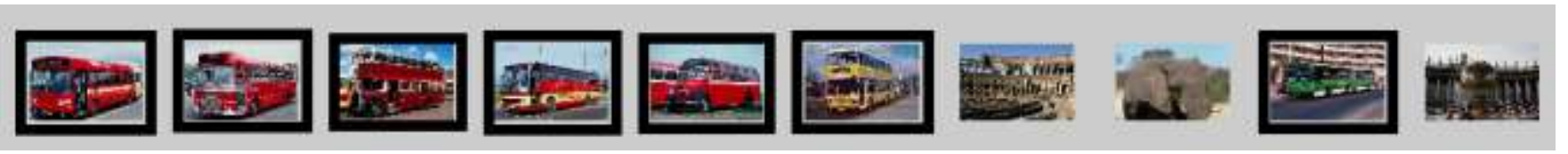

4.d.First Ten Retrieved images using RGB-Haar Wavelets-Level-5 image retrieval technique (Total Relevant retrieved images=07)

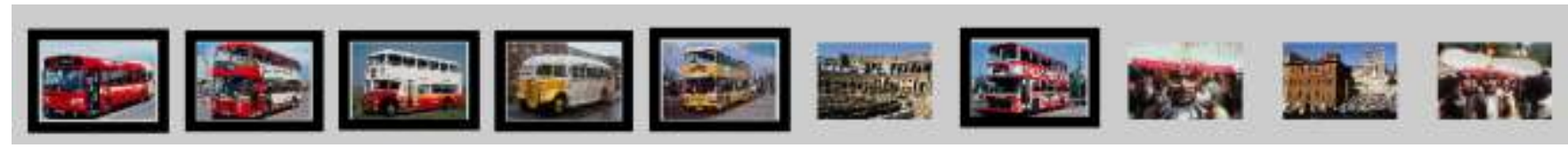

4.e.First Ten Retrieved images using RGB-Haar Wavelets-Level-7 image retrieval technique (Total Relevant retrieved images=06)

Figure. 4. First Ten Retrieved Images for the given Query Image using Discussed Image Retrieval Techniques.

[All result images are sequentially ordered from left to right with respect to their degree of matching with query image. Here total number of relevant retrieved images is higher in RGB-Haar Wavelets-Level-5 based image retrieval technique as compared to other techniques. Higher level Haar Wavelets are giving very coarse texture features while lower level Haar Wavelets are giving too fine texture features losing discrimination capability, which is not really useful in image retrieval. Hence Haar Wavelets-Level-5 give better discrimination power for image retrieval.] 


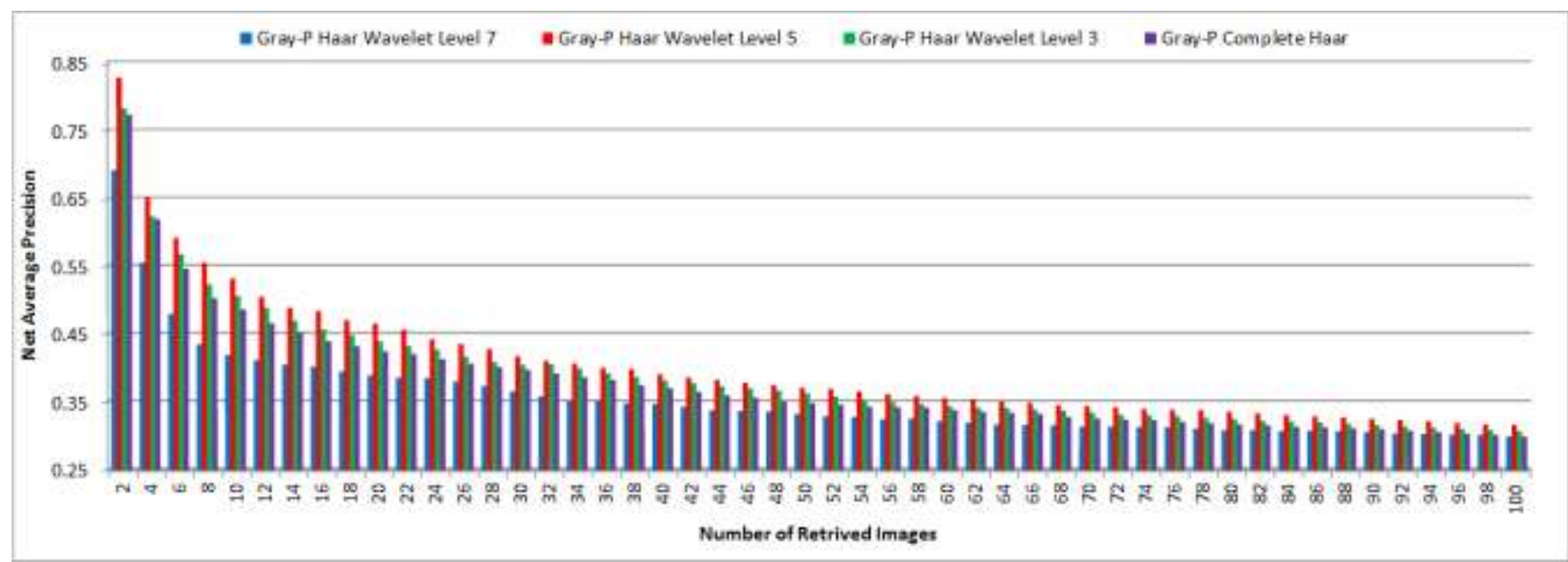

5.a. Net Average Precision Vs Number of Retrieved Images for Complete Haar and Gray-Haar Wavelets (Gray-P)

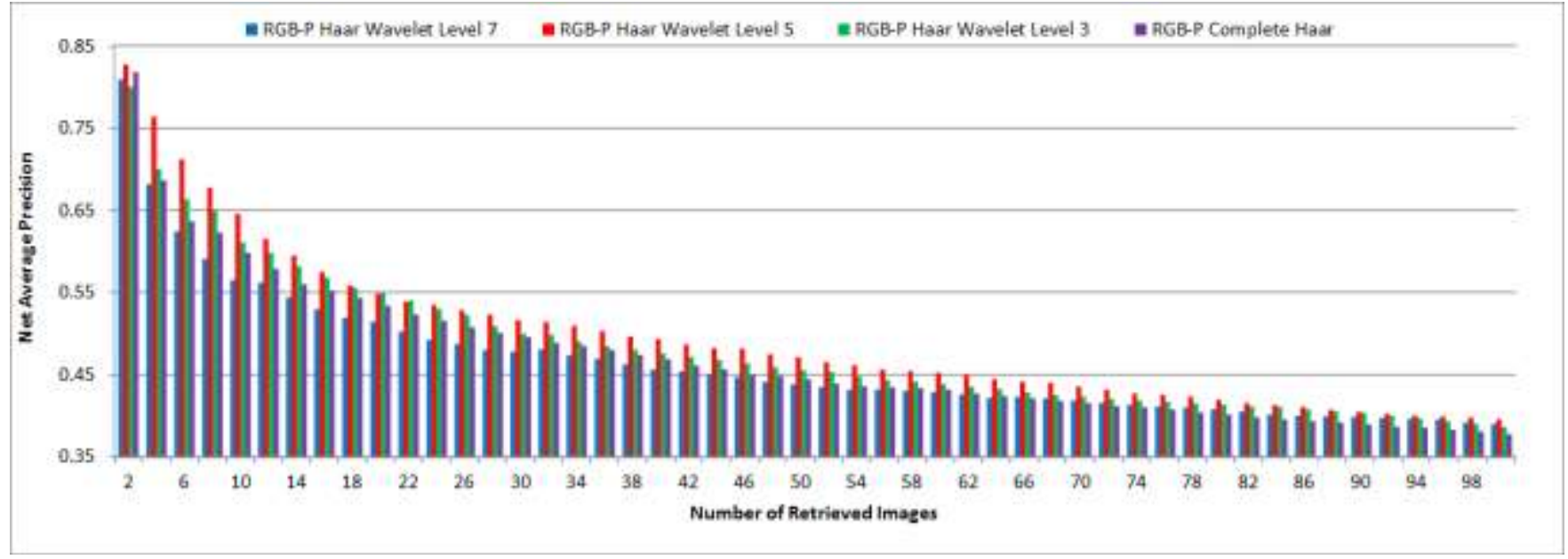

5.b. Net Average Precision Vs Number of Retrieved Images for Complete Haar and RGB-Haar Wavelets (RGB-P)

Figure. 5. Net Average Precision Vs Number of Retrieved Images for Complete Haar and Haar Wavelets of level 3,5,7 [Here Haar Waveletsare outperforming complete Haar in case of precision. The Precision/Recall values of Color Haar Wavelets(RGB) are higher than those of Gray-Haar Wavelets. The precision values of Haar Waveletslevel-5 are higher than all other Haar Wavelets and even than the complete Haar based CBIR]

Figure 6 shows the net average recall plotted against number of retrieved images for complete Haar and Gray/Color-Haar Wavelets based CBIR for various levels of Haar Wavelets. Here Haar Wavelets are performing better than complete Haar T. in case of Recall. The Recall values of RGB-Haar Wavelets shown in figure 6.b are higher than those of Gray-Haar Wavelets shown in figure 6.a. The recall values of Haar Wavelets level-5 are slightly higher than all other Haar Wavelets and even than the complete Haar T. based CBIR.

The crossover point of precision and recall of CBIR techniques acts as one of the important parameters to judge performance. Figure 7 gives the crossover of precision and recall plotted against Number of retrieved images for Gray-Haar Wavelets and Color-
Haar Wavelets compared with Complete Haar T. Figure 7.a shows crossover of precision and recall for Gray-Haar Wavelets and figure 7.b shows it for Color-Haar Wavelets. Here the crossover point of Haar Wavelet level-5 is the highest (0.32 in Gray-Haar Wavelet and 0.401 in Color-Haar Wavelet) showing the best performance of CBIR as compared to complete Haar T. based CBIR and Haar Wavelets of other levels. Overall the crossover point values in Color-Haar Wavelets are greater than the respective crossover point values in Gray-Haar Wavelets. The precision/recall values and crossover points proves that the discrimination capability of Haar Wavelets in CBIR is better than complete Haar based CBIR and Haar Wavelets of level 5 gives the best performance in all. 


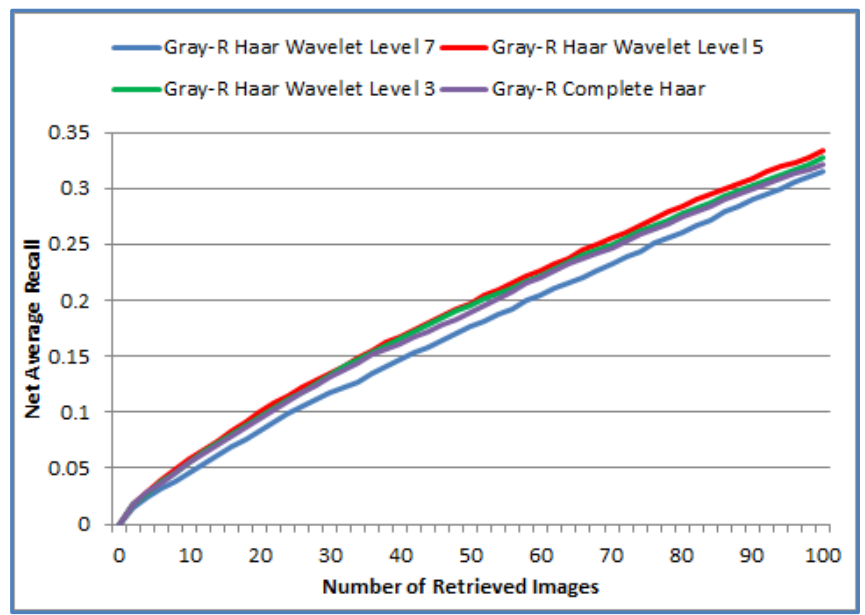

6.a.Net Average Recall for Gray-HaarWavelet (Gray-R)

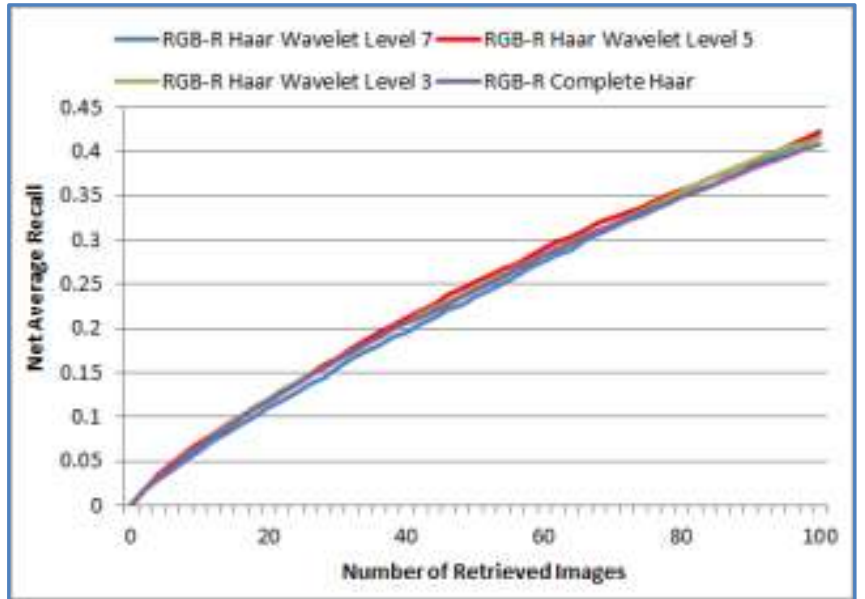

6.b.Net Average Recall for Color-Haar Wavelet (RGB-R)

Figure 6. Net Average Recall v/s Number of Retrieved Images for Complete Haar transform and Haar Wavelets of level 3,5,7.

[Here recall for Color-Haar Wavelets based CBIR is higher than that of Gray-HaarWavelet based CBIR. The Recall values of Haar Waveletslevel-5 are marginally higher than the other Haar wavelet levels]
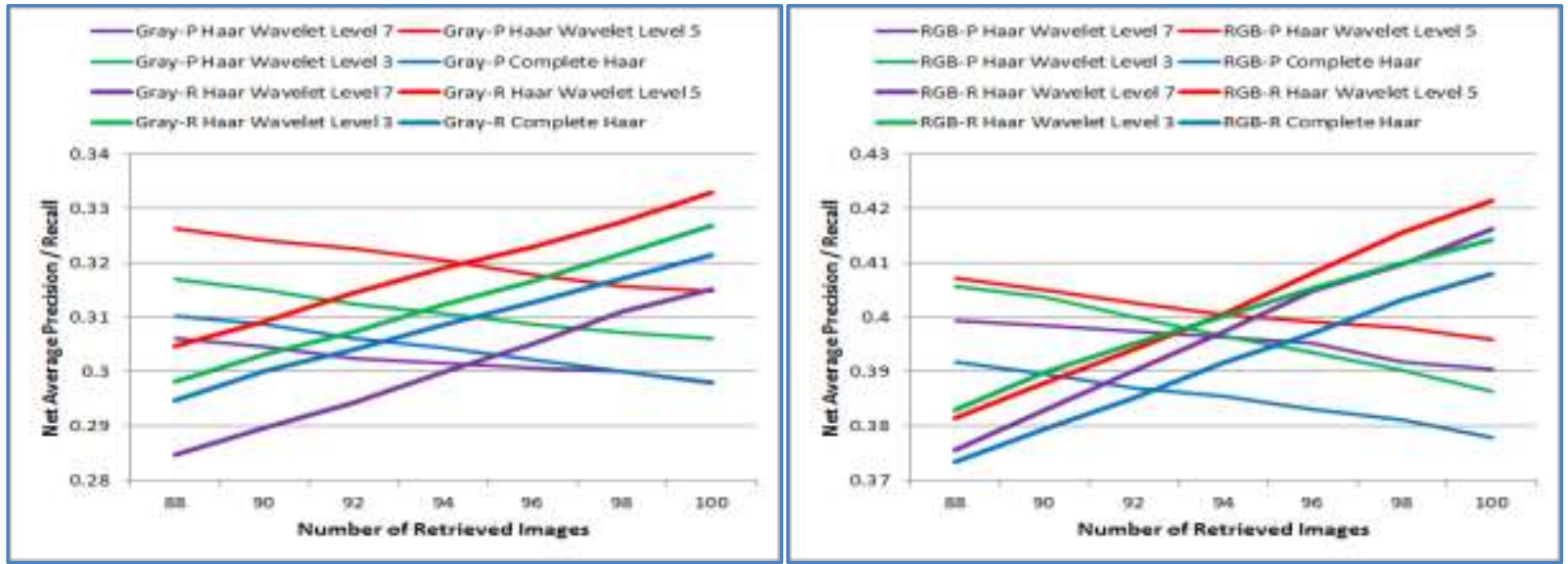

7.a.Crossover of Precision-Recall for Gray-Haar Wavelet (Gray-P/R) 7.b. Crossover of Precision-Recall for Color-Haar Wavelet(RGB-P/R)

Figure 7. Crossover Points of Precision-Recall in plot of Net Average Precision/Racall Vs Number of Retrieved Images for Complete Haar and Haar Wavelet of level 3,5,7.

[Here the crossover point values of Haar Wavelets level-5 are highest in both Color-Haar Wavelets based CBIR as well as in Gray-Haar Wavelets based CBIR. So as the performance of Haar Wavelets level-5 based CBIR are higher as it gives better discrimination capability in image retrieval with respect to color-texture features.] 


\section{CONCLUSION}

More precise and faster retrieval techniques are needed to access the large image archives being generated, for finding similar images.

Here in this paper the Wavelet Pyramid based image retrieval techniques [1] have been proposed using Haar transform. The proposed Gray-Haar Wavelet and Color-Haar Wavelet based CBIR techniques are tested using 55 queries fired on the image database with 1000 images spread over 11 categories. The precision/recall values show that Haar wavelet outperforms complete Haar $\mathrm{T}$. based image retrieval, proving that Haar Wavelets have better discrimination capability. Also Haar Wavelet of level-5 is giving the best performance than other Haar Wavelets because higher level Haar Wavelets are giving very coarse texture features while lower level Haar Wavelet are giving too fine texture features losing discrimination capability, which are not really useful in image retrieval and proper color-texture properties are carved by Haar wavelet level-5 at greatly reduced query execution time.

\section{REFERENCES}

[1] H.B.Kekre, Sudeep D. Thepade, "Image Retrieval using Color-Texture Features Extracted from Walshlet Pyramid", ICGST International Journal on Graphics, Vision and Image Processing (GVIP), Volume 10, Issue I, Feb.2010, pp.9-18, Available online www.icgst.com/gvip/Volume10/Issue1/P1150938876.html.

[2] H.B.Kekre, Sudeep D. Thepade, "Image Retrieval using Augmented Block Truncation Coding Techniques", Proc. ACM Int. Conf. on Advances in Computing, Communication and Control (ICAC3-2009), 23-24 Jan 2009, Fr. Conceicao Rodrigous College of Engg., Mumbai. Is uploaded and available online at ACM portal.

[3] H.B.Kekre, Sudeep D. Thepade, "Rendering Futuristic Image Retrieval System", Proc. National Conf. on Enhancements in Computer, Comm. and Info. Technology, EC2IT-2009,20-21 Mar 2009, K.J. Somaiya COE, Vidyavihar, Mumbai-77.

[4] H.B.Kekre, Sudeep D. Thepade, "Color Traits Transfer to Grayscale Images", IEEE -International Conference on Emerging Trends in Engineering and Technology, ICETET2008, 16-18 July 2008, Raisoni College of Engineering, Nagpur. Is uploaded and available online at IEEE Xplore CSDL, ACM Portal

[5] H.B.Kekre, Sudeep D. Thepade, "Image Blending In Vista Creation using Kekre's LUV Color Space" , SPIT -IEEE Colloquium and International Conference, 04-05 Feb 2008 ,SPIT Andheri ,Mumbai.

[6] K.-C. Liang and C. C. Kuo, "WaveGuide: A Joint WaveletBased Image Representation and Description System," IEEE Trans. on ImageProcessing, vol. 8, no. 11, pp.1619-1629, 1999

[7] W. Y. Ma, B. S. Manjunath, "A comparison of wavelet features for texture annotation," Proc. of IEEE Int. Conf. on Image Processing, Vol. II, pp. 256-259, Washington D.C., Oct. 1995.

[8] Hirata K. and Kato T. "Query by visual example - contentbased image retrieval", In Proc. of Third International
Conference on Extending Database Technology, EDBT'92, 1992, pp 56-71.

[9] Haar, Alfred, "Zur Theorie der orthogonalen Funktionen systeme". (German), Mathematische Annalen, volume 69, No. 3, 1910, pp. 331-371.

[10] Charles K. Chui, “An Introduction to Wavelets”, Academic Press, 1992, San Diego, ISBN 0585470901.

[11] http://wang.ist.psu.edu/docs/related/Image.orig (last referred on June, $10^{\text {th }}, 2009$ )

[12] A. K. Jain , A. Vailaya, "Image Retrieval using Colour and Shape," In Proc. of 2nd Asian Conference on Computer Vision (ACCV-95), Singapore, 1995, pp. 529-533.

[13] H.B.Kekre, Sudeep D. Thepade, "Ubicomp The Future of Computing Technology", TechnoPath : Journal of Science Technology and Management, Volume 1, Issue 2, 2009.

[14] Robert Li, Jung Kim, "Image Compression Using Fast Transformed Vector Quantization", IEEE Applied Imagery Pattern Recognition Workshop, 2000 Proceedings, Volume 29, 2000, pp. $141-145$.

[15] B.G.Prasad, K.K. Biswas, and S. K. Gupta, "Region -based image retrieval using integrated color, shape, and location index", International Journal on Computer Vision and Image Understanding Special Issue: Colour for Image Indexing and Retrieval, Volume 94, Issues 1-3, April-June 2004, pp.193233.

[16] Minh N. Do, , and Martin Vetterli, , "Wavelet-Based Texture Retrieval Using Generalized Gaussian Density and KullbackLeibler Distance", IEEE Transactions On Image Processing, Volume 11, Number 2, pp.146-158, February 2002.

[17] A. Gupta, R. Bach, C. Fuller, A. Hampapur, B. Horowitz, R. Jain, C.F. Shu "The Virage image search engine: an open framework for image management" in Storage and Retrieval for Image and Video Databases IV, Proc SPIE Vol. 2670, pp 76-87, 1996.

[18] M. Flickner, H. Sawhney, W. Niblack, J. Ashley, Q. Huang, B. Dom, M. Gorkani, J. Hafner, D. Lee, D. Petkovic, D. Steele, and P. Yanker. "Query by image and video content: The QBIC system," IEEE Computer, vol. 28, pp. 23-32, 1995.

[19] H.B.Kekre, Sudeep D. Thepade, "Boosting Block Truncation Coding using Kekre's LUV Color Space for Image Retrieval", WASET International Journal of Electrical, Computer and System Engineering (IJECSE), Volume 2, Number 3, Summer 2008. Available online at http://www.waset.org/ijecse/v2/v2-3-23.pdf

[20] M. La Cascia, S. Sethi, S. Sclaroff. "Combining textual and visual cues for content-based image retrieval on the world wide web", In IEEE Workshop on Content-based Access of Image and Video Libraries, pp 24-28, Santa Barbara, CA, June 1998.

[21] Carson M. Thomas, S. Belongie, J. M. Hellerstein, and J. Malik, "Blobworld: a system for region-based image indexing and retrieval", In Visual Information and Information Systems (VISUAL), LNCS 1614, pages 509516, Amsterdam, The Netherlands, June 1999. 
[22] S. Sclaroff, L. Taycher, and M. La Cascia, "ImageRover: a content-based image browser for the world wide web", In IEEE Workshop on Content-based Access of Image and Video Libraries, pages 2-9, San Juan, Puerto Rico, June 1997.

[23] H.B.Kekre, Tanuja K. Sarode, Sudeep D. Thepade, "Image Retrieval using Color-Texture Features from DCT on VQ Codevectors obtained by Kekre's Fast Codebook Generation", ICGST International Journal on Graphics, Vision and Image Processing (GVIP), Volume 9, Issue V, Sept. 2009, pp. 1-8, Available onlineathttp://www.icgst.com/gvip/Volume9/Issue5

[24] Zaher Al Aghbari and Ruba Al-Haj, "Building SSeg-Tree for Image Representation and Retrieval", ICGST Int. Journal on Graphics, Vision and Image Processing (GVIP), Special Issue on Image Retrieval and Representation, Vol. 6, Year 2006, pp. 101-109.

[25] M.Eisa, I.Elhenawy, A.E.Elalafi and H. Burkhardt, "Image Retrieval based on Invariant Features and Histogram Refinement", ICGST Int. Journal on Graphics, Vision and Image Processing (GVIP), Special Issue on Image Retrieval and Representation, Vol. 6, Year 2006, pp. 7-11.

[26] H.B.Kekre, Sudeep D. Thepade, "Color Based Image Retrieval using Amendment Block Truncation Coding with YCbCr Color Space”, International Journal on Imaging (IJI), Volume 2, Number A09, Autumn 2009, pp.2-14. Available online at www.ceser.res.in/iji.html

[27] H.B.kekre, Tanuja K. Sarode, Sudeep D. Thepade, "ColorTexture Feature based Image Retrieval using DCT applied on Kekre's Median Codebook", International Journal on Imaging (IJI), Volume 2, Number A09, Autumn 2009,pp. 5565. Available online at www.ceser.res.in/iji.html

[28] Tuceryan M., Jain A.K.,"Texture Analysis Handbook of Pattern Recognition and Computer Vision (Eds. C.H.Chen, L.F.pau, P.S.P.Wang), 1994.

\section{Author Biographies}

Dr. H. B. Kekre has received B.E. (Hons.) in Telecomm. Engineering. from Jabalpur University in 1958, M.Tech (Industrial Electronics) from IIT Bombay in 1960, M.S.Engg. (Electrical Engg.) from University of Ottawa in 1965 and Ph.D. (System Identification) from IIT Bombay in 1970.
He has worked as Faculty of Electrical Engg. and then HOD Computer Science and Engg. at IIT Bombay. For 13 years he was working as a professor and head in the Department of Computer Engg. atThadomalShahani Engineering. College, Mumbai. Now he is Senior Professor at MPSTME, SVKM's NMIMSUniversity. He has guided 17 Ph.Ds, more than 100 M.E./M.Tech and several B.E./B.Tech projects. His areas of interest are Digital Signal processing, Image Processing and Computer Networking. He has more than 300 papers in National / International Conferences and Journals to his credit. $\mathrm{He}$ was Senior Member of IEEE. Presently He is Fellow of IETE and Life Member of ISTE Recently nine students working under his guidance have received best paper awards. Currently 10 research scholars are pursuing $\mathrm{Ph} . \mathrm{D}$. program under his guidance.

Sudeep D. Thepade has Received B.E.(Computer) degree from North Maharashtra University with Distinction in 2003. M.E. in Computer Engineering from University of Mumbai in 2008 with Distinction, currently pursuing Ph.D. from SVKM's NMIMS, Mumbai. He has about than 07 years of experience in teaching and industry. He was Lecturer in Dept. of Information Technology at Thadomal Shahani Engineering College, Bandra(w), Mumbai for nearly 04 years. Currently working as Associate Professor in Computer Engineering at Mukesh Patel School of Technology Management and Engineering, SVKM's NMIMS University, Vile Parle(w), Mumbai, INDIA. He is member of International Association of Engineers (IAENG) and International Association of Computer Science and Information Technology (IACSIT), Singapore. His areas of interest are Image Processing and Computer Networks. He has about 75 papers in National/International Conferences/Journals to his credit with a Best Paper Award at International Conference SSPCCIN-2008, Second Best Paper Award at ThinkQuest-2009 National Level paper presentation competition for faculty and Best Paper Award at Springer International Conference ICCCT-2010.

Akshay Maloo is currently pursuing B.Tech. (CS) from MPSTME, NMIMS University, Mumbai. His areas of interest are Artificial intelligence, Image Processing, Computer Networks and Security. He has 9 papers in National/International Conferences/Journals to his credit. 